\title{
PERTINENCIA DE LA FORMACIÓN A LOS CAPACITADORES \\ ELECTORALES EN MATERIA DE LOS MÉTODOS DE SOLUCIÓN DE CONFLICTOS PARA GENERAR DE FORMA CREATIVA SOLUCIONES A LOS CONFLICTOS ELECTORALES ${ }^{1}$
}

\section{THE IMPORTANCE OF THE KNOWLEDGE OF ALTERNATIVE DISPUTE RESOLUTIONS BY THE ELECTOR TRAINERS IN ORDER TO BUILD CREATIVE WAYS TO SOLVE ELECTORAL CONFLICTS}

Jéssica Marisol Vera

Doctora por la Universidad Autónoma de Nuevo León, Doctorado en Filosofía con Acentuación en Ciencias Políticas, Profesora investigadora de la UANL, México. E-mail: jessica.vera@gmail.com

Eni Valdes-Espinosa

Maestría en Educación Southern New Hamshire University, Maestría en Clínica Psicoanalítica Universidad Autónoma de Nuevo León, estudiante de Doctorado de Métodos Alternos de Solución de Conflictos, Profesora UANL, Psicoanalista. México. E-mail: eni_valdes@hotmail.com

RESUMEN: Las crisis electorales tienen el potencial de causar un daño significativo a la economía nacional y medios de vida individuales; resultar en violencia significativa, desplazamiento y pérdida de propiedad; y en el peor de los casos conducir a una guerra civil o la fragmentación del estado a lo largo líneas étnicas o políticas. Las crisis electorales pueden ser más probables en entornos de transición y en países con líneas de falla basadas en identidad profunda o una historia marcada por disturbios y conflicto. En estas situaciones, se puede recurrir al mediador no solo para facilitar las conversaciones sino también para crear espacios de prevención y capacitación, cómo se desarrollará en este artículo.

\footnotetext{
${ }^{1}$ Artigo recebido em 10/12/2020, sob dispensa de revisão.
} 
PALABRAS CLAVE: Conflictos electorales; mediación; capacitación.

\begin{abstract}
The electoral crisis have the potential to generate great damages to national economy and private lives, violence, property withdrawal and lead, in the worse scenario, to a civil war or to a state fragmentation according to ethnical or political ideologies. The electoral crisis are more frequent in transition environments and the countries with fault lines based on deep identity or a history marked by conflicts. In this situations, the mediator may deal with these conflicts not only to easy the conversations but also to create prevention and training spaces, what will be studied in this article.
\end{abstract}

KEY WORDS: electoral conflicts; mediation; training.

\title{
1. Introducción
}

En los últimos 30 años, un mayor número de países ha empleado elecciones para determinar la voluntad de su gente y formar gobiernos representativos y democráticos. Algunos actores internacionales, incluida la Asamblea General de las Naciones Unidas, acogió con beneplácito esto por contribuir a la paz y la estabilidad nacional y, por ende, internacionales. Al mismo tiempo, esta tendencia positiva trae ciertos desafíos. Estos incluyen escenarios donde una circunscripción política importante no acepta el resultado del proceso electoral.

En este sentido aparecen nuevas estrategias de prevención de este tipo de conflictos que generan crisis electorales. El objetivo de estas estrategias es promover los votos aceptados y generalmente pacíficos e integra enfoques, prácticas y mecanismos para resolver conflictos entre candidatos y partidos en la maquinaria electoral en sí (CICIE, 2015).

Aunque todavía es relativamente poco común, estos eventos a menudo tienen consecuencias significativas. Las crisis electorales tienen el potencial de causar un daño significativo a la economía nacional y medios de vida individuales; resultar en violencia significativa, desplazamiento y pérdida de propiedad; y en el peor de los casos conducir a una guerra civil o la fragmentación del estado a lo largo líneas étnicas o políticas. Las crisis electorales pueden ser más probables en entornos de transición y en países con líneas de falla 
basadas en identidad profunda o una historia marcada por disturbios y conflicto (Kane \& Hayson, 2016).

En estas situaciones, se puede recurrir al mediador no solo para facilitar las conversaciones sino también para crear espacios de prevención y capacitación.

\section{Capacitadores electorales}

El siglo XXI, de manera particular en esta segunda década, se ha visto el declive de la confianza ciudadana en los actores políticos. La realidad actual ha generado un caldo de cultivo de apatía política y hartazgo ciudadano, pues se han presentado casos de corrupción sin precedentes que parecen haber tenido un efecto corrosivo en las democracias latinoamericanas y por supuesto en el sistema político mexicano.

Por lo que es importante hablar de la capacitación de las estructuras como algo vital para fomentar el aprendizaje continuo, la actualización profesional y la efectividad de cada integrante en los cargos que ocupa tanto dentro del partido como también los que pudiese obtener en la Administración o en el seguimiento de elecciones. Sin embargo, esta capacitación de las estructuras partidarias no debe sujetarse solo al período electoral, sino que debe darse también durante la gestión de un gobierno de manera preventiva a los conflictos que se puedan suscitar. Pues, en efecto, como lo menciona Thompson (2012). La participación política reclama el ejercicio de derechos antes, durante y después de un proceso electoral específico. Estos derechos se encuentran resguardados por los ordenamientos jurídicos y el eje de formación política está incorporado en ellos, y adquiere cada vez mayor relevancia (Chacon, 2018).

Por lo que es importante hablar de la importancia de incluir dentro de los presupuestos de los partidos capacitaciones que permitan a sus miembros y participantes del proceso electoral emplear medidas preventivas y modelos de mediación en crisis electorales. En años de elecciones federales, los partidos reciben financiamiento adicional para gastos de campaña, el cual equivale a otro tanto similar al que reciben cada daño para actividades ordinarias permanentes. Además, los partidos reciben apoyos para actividades específicas de educación y capacitación política, también para producir programas de radio y televisión (Aparicio, 2006). 
Por lo tanto, si es considerado el monto total del financiamiento público que se dedica a los procesos electorales federales en su conjunto resulta que la democracia mexicana es de las más costosas del mundo. De acuerdo con el estudio hecho por Valdez y Huerta (2018) tan sólo en el año 2017, un año no electoral, se aprobó por la Cámara de Diputados un presupuesto de 10 mil 932 millones 449 mil 787 pesos, de los cuales 4 mil 138 millones de pesos está destinado a financiar a los nueve partidos políticos.

En la siguiente tabla se muestra el presupuesto asignado a los partidos políticos en el año de 2017 de acuerdo a datos de

Presupuesto asignado a los partidos políticos

\begin{tabular}{|c|c|c|c|c|c|}
\hline & $\begin{array}{l}\text { Actividades } \\
\text { ordinarias } \\
\text { permanentes }\end{array}$ & $\begin{array}{c}\text { Educación, capacitación, } \\
\text { investigación } \\
\text { socioeconómica y } \\
\text { politica, y tareas } \\
\text { editoriales }\end{array}$ & $\begin{array}{l}\text { Franquicias } \\
\text { postales }\end{array}$ & $\begin{array}{l}\text { Franquicias } \\
\text { telegráficas }\end{array}$ & Total por partido \\
\hline Partido Acción Nacional & $\$ 759,442,113$ & $\$ 22,783,264$ & $\$ 8,757,743$ & $\$ 77,055$ & $\$ 791,060,175$ \\
\hline $\begin{array}{l}\text { Partido Revolucionario } \\
\text { Institucional }\end{array}$ & $\$ 1,004,337,987$ & $\$ 30,130,140$ & $\$ 8,757,743$ & $\$ 77,055$ & $\$ 1,043,302,925$ \\
\hline $\begin{array}{l}\text { Partido de la Revolución } \\
\text { Democrática }\end{array}$ & $\$ 455,159,108$ & $\$ 13,654,773$ & $\$ 8,757,743$ & $\$ 77,055$ & $\$ 477,648,679$ \\
\hline Partido del Trabajo & $\$ 217,254,999$ & $\$ 6,517,650$ & $\$ 8,757,743$ & $\$ 77.055$ & $\$ 232,607,447$ \\
\hline $\begin{array}{l}\text { Partido Verde } \\
\text { Ecologista de México }\end{array}$ & $\$ 338,022,381$ & $\$ 10,140,671$ & $\$ 8,757,743$ & $\$ 77,055$ & $\$ 356,997,830$ \\
\hline Movimiento Ciudadano & $\$ 313,331,759$ & $\$ 9,399,953$ & $\$ 8,757,743$ & $\$ 77,055$ & $\$ 331,566,510$ \\
\hline Nueva Alianza & $\$ 242,637,017$ & $\$ 7,279,110$ & $\$ 8,757,743$ & $\$ 77,055$ & $\$ 258,750,925$ \\
\hline Morena & $\$ 380,596,946$ & $\$ 11,417,908$ & $\$ 8,757,743$ & $\$ 77,055$ & $\$ 400,849,652$ \\
\hline \multirow[t]{2}{*}{ Encuentro Social } & $\$ 230,202,064$ & $\$ 6,906,062$ & $\$ 8,757,743$ & $\$ 77,055$ & $\$ 245,942,944$ \\
\hline & $\$ 3,940,984,374$ & $\$ 118,229,531$ & $\$ 78,819,687$ & $\$ 693,495$ & $\$ 4,138,727,087$ \\
\hline
\end{tabular}

Fuente: Valdez y Huerta, 2018.

Como respuesta a estos cuestionamientos, se podría pensar que más que un gasto el presupuesto destinado a los procesos electores, representa una inversión orientado a afianzar el proceso de cambio y democratización en México, así como institucionalizar y fortalecer el sistema de partidos políticos, motivando una mayor participación ciudadana y reducir el abstencionismo (Valdez \& Huerta, 2018).

\section{Conflictos en periodos de elección}

Las brazas de una crisis electoral suele ser un supuesto defecto en el proceso electoral, generalmente relacionado con reclamos de manipulación sistemática de preparaciones electorales, generalizado fraude el día de la elección, o serias irregularidades en el conteo de resultados (Kane \& Hayson, 2016). Puede agravarse por una supuesta falta de confianza en la imparcialidad de la elección comisión y probabilidad de que haya un manejo transparente e independiente de quejas de fraude y otras malversaciones. 
Los conflictos relacionados con las elecciones pueden tener lugar durante todos los puntos críticos del ciclo electoral, aunque los momentos más volátiles propensos a conflictos son durante la elección y las fases postelectorales. Mientras que los períodos preelectorales son en gran medida sin problemas, hay una tendencia notable en el proceso de las elecciones Las disputas se intensifican en el período postelectoral.

Estas disputas electorales (incluyendo no aceptación de los resultados electorales) a menudo van acompañados de violencia, que socava los esfuerzos regionales y continentales para lograr la paz y la prosperidad. La violencia a menudo tiene lugar en el contexto de personas débiles o inexistentes mecanismos de gestión de conflictos (Shale \& Gerenge, 2017).

La mediación electoral es, por lo tanto, un mecanismo para tratar disputas relacionadas con las elecciones durante El ciclo electoral. Con base en esto, afirmamos que contrario a lo convencional En la práctica, la mediación de las disputas electorales no es exclusiva de partidos políticos u organismos estatutarios, pero también deberían involucrar a una sociedad civil más amplia. Eso debe ser un esfuerzo de colaboración entre los organismos de gestión electoral, partidos políticos y todos los demás interesados con las habilidades para contribuir a la mitigación del conflicto (UNPD, 2009).

El desafío que enfrenta el mediador cuando se enfrenta a tales crisis es más matizado que simplemente determinar si el fraude se cometió de hecho. Más bien es tratar de distinguir entre elecciones que tuvieron fraude u otra mala conducta (no es raro en países con instituciones débiles o en entornos posteriores a conflictos) y fraudulentos elección con credibilidad limitada o nula. El primero admite la posibilidad de abordar irregularidades electorales a través de remedios técnicos mientras que el segundo no (Shale \& Gerenge, 2017).

En el caso de México el largo proceso de transformación de las instituciones políticas electorales tenía entre sus objetivos implícitos reducir el número y la importancia de conflictos derivados de elecciones y hacerlas confiables; ello aseguraría la gobernabilidad, pero, además, la confianza general en las instituciones de la democracia. En caso de disconformidad con los resultados, los mecanismos legales aprobados permitirían tomar decisiones que no dejarían ninguna duda sobre la validez de esos desenlaces; no obstante, se han presentado cuestionamientos de todo tipo a los procesos y a los resultados y, en algunos 
casos, se llegó a enfrentamientos violentos, con consecuencias de muertos y heridos (Nassif, 2007).

En México es común escuchar del conflicto en los momentos de elecciones y post elecciones, se escucha hablar incluso de violencia política. Por lo que es importante la búsqueda de distintos mecanismos alternos para la solución de conflictos en materia electoral, a continuación, se presentan estos mecanismos y su forma de emplearlos, así como la importancia de la capacitación.

\section{MSC como alternativa para solucionar los conflictos electorales}

Los MSC se han posicionado en la actualidad como un espacio para poder atender diversas circunstancias que las partes en su momento no encontraron una alternativa acorde para poder resolver su conflictiva. Esta alternativa pacífica ha venido a despresurizar al sistema de justicia y al litigio, sin embrago, los MSC tienen muchos otros beneficios que se han venido estudiando a través del tiempo. Dentro de los MSC encontramos mediación, arbitraje, negociación y conciliación, cada uno de ellos desde su lógica y metodología científica y jurídica aportan a la cultura de la paz. Si bien en México, los MSC se posicionan a partir de 1994 en la constitución de $1917^{2}$ se le conminaba al juez a conciliar entre las partes. Como ya mencionamos los beneficios son múltiples (Hernandez, G. Cabello, P. , 2017), uno de estos beneficios que nos brindan los MSC es la paz la cual ha sido definida de muchas maneras. Pérez \& Gardey (2013) plantean que visto como un estado positivo es un estado de tranquilidad y quietud y visto desde un sentido negativo es la ausencia de guerra.

Hace años se consideraba que la paz social era el fin último de las leyes (Arango Durling, 2007), hoy en día va más allá de un fin, se entiende como un elemento sine qua non de aquellos sistemas judiciales modernos.

Es por ello por lo que consideramos que la paz requiere de una participación de la sociedad, de acciones de paz que busquen la justicia y la equidad de propuestas de paz que cuiden los intereses de las personas que están en conflicto, basándose en la sensibilidad, la pasión, la conciencia, la paciencia y la bondad. Lo anterior se puede lograr mediante el uso de los MSC puesto que estos fomentan que sea la sociedad quienes participen directamente en la solución de sus propios conflictos. Se busca que, mediante la mediación, la

\footnotetext{
${ }^{2}$ Constitución de los Estados Unidos Mexicanos
} 
negociación, la conciliación y el arbitraje se puedan prevenir, solucionar los conflictos, así como también se logre una ciudadanización de la justicia.

Miembros del claustro académico del doctorado en Métodos Alternos de Solución de Conflictos de la Facultad de Derecho y Criminología de la Universidad Autónoma de Nuevo León, trabajan hacia los MSC como herramientas de paz, mencionaremos algunos de ellos a continuación:

El Dr. Francisco Javier Gorjón Gómez (2011) plantea que los MSC como herramientas de paz son el camino para tener una cultura de la no violencia y la solución de conflictos. Considera que los MSC representan para la paz el soporte legal. La misión de los MSC es desarrollar esquemas de justicia y paz en cualquier tipo conflictos. Su finalidad es lograr que las partes descubran maneras de autocomposición apoyada en sus intereses y necesidades.

Gorjón plantea tres puntos de intersección entre la paz y los MASC en donde ambos operan en una misma dirección:

1. La promoción del arreglo pacífico de los conflictos, el respeto y el entendimiento mutuo y la cooperación internacional.

2. La posibilidad en que todas las personas en todos los niveles desarrollen aptitudes para el diálogo, la negociación, la formación de consensos y la solución pacífica de conflictos, a través de la mediación, la conciliación y el arbitraje.

3. Desarrolla competencias para que la sociedad acceda a una vida más justa conforme a la Declaración sobre una Cultura de Paz de las Naciones Unidas, así como a los principios y directrices de las cátedras UNESCO a través del conocimiento, la investigación, la docencia y la cooperación.

El autor considera que la mejor manera para que perduren las acciones de paz es mediante la educación multidimensional asociada a los MSC y menciona tres grupos de medidas para lograrlo:

1. Promoviendo y reforzando las actividades de educación, la capacitación para promover el diálogo, el consenso y la solución de conflictos.

2. Reforzando las actividades en marcha de las entidades pertinentes del sistema de las naciones unidas que tienen como finalidad capacitar y educar en la prevención 
de los conflictos y la gestión de la crisis, el arreglo pacífico de los conflictos y la solidificación de la paz.

3. Extender las iniciativas que favorecen la cultura de paz realizadas por instituciones mundiales de enseñanza superior, así como también el proyecto al programa de Universidades Gemelas y de Cátedra UNESCO.

Uno de los aportes que Dr. Gorjón (2017) ha realizado a los MSC es el desarrollo de la teoría de los Intangibles de los MSC, compuesto por 47 intangibles con la intención de promover que la sociedad conozca y entienda su aplicación. Los intangibles se centran en identificar el valor real y operativo de la negociación, la mediación, la conciliación y el arbitraje siendo su principal actor el capital intelectual el cual se desagrega en el capital humano representado por los operadores de los MSC, el capital estructural representado por los administrativos que proporcionan la estabilidad y por el capital relacional siendo estos los receptores y los usuarios (Gorjón Gómez F. J., 2016).

El Dr. Paris Alejandro Cabello (2016) plantea que la mediación es un método alternativo para la transformación de los conflictos y una vía hacia la construcción de una cultura de paz. Es por ello por lo que la mediación es considerada una herramienta de paz que ayuda a crear un entorno pacífico, al propiciar la transformación de los conflictos en ideas, fomentando el diálogo, la empatía, la solidaridad, la no-violencia, la comprensión, la integración, la creatividad, la participación y el consenso, los cuales son elementos de carácter sine qua non de la paz.

Paris plantea que la mediación es una vía para alcanzar la paz por el hecho de ser transformadora de conflictos al trascender los objetivos de las partes en conflictos fijando otros objetivos. La cultura de paz y la mediación comparten características básicas como el fomento de la tolerancia, la cooperación, la justicia, la empatía, la democracia, la solidaridad, el arreglo pacífico. la equidad, la participación de la sociedad, el arreglo pacífico de los conflictos (Cabello Tijerina, 2017).

$\mathrm{El}$ autor considera que los MSC fomentan que las partes tengan un rol activo, que hablen y se escuchen, que sean ellos mismos los protagonistas en el proceso. Los MSC al promover valores a lo largo del proceso, fomentan a su vez la construcción y el mantenimiento de la cultura de paz. 
Para lograr que la sociedad sea la protagonista en el logro de la paz se requiere que sea ella misma la que desarrolle habilidades, aptitudes y capacidades para el manejo de conflictos, la formación de consensos y el dialogo. La mediación permite construir este cambio cultural y social basándose en una educación de conocimientos, de valores y de resolución de conflictos. Los MSC ayudan a construir sociedades con un desarrollo social, favorece el decremento de desigualdades sociales y económicas. Buscando establecer de esta manera una cultura de paz donde se respete la igualdad de género y los derechos humanos, así como que se promueva la participación y factores políticos, culturales y socioestructurales (Cabello Tijerina, 2016).

Destacamos uno de los aportes realizados por Dr. Paris a los MSC, es la creación de la Teoría de la irenología de los MSC, en la cual plantea buscar una cultura de paz, estableciendo ejes rectores de actuación que busquen soluciones a la impetración de la justicia, que estudie el conflicto y su multidimensionalidad con la intención de brindar alternativas científicas buscando que se genere un cambio en las culturas violentas por culturas que sean más participativas, democráticas y pacíficas (Cabello Tijerina, 2015).

La Dra. Reyna Lizeth Vázquez Gutiérrez (2015) plantea como la mediación educativa es una herramienta de educación para la paz y cultura de paz. En donde familia, escuela y sociedad son los tres agentes que constituyen al ser social, la Dra. Vázquez plantea que la escuela cuenta con los elementos necesarios para la interiorización de habilidades, actitudes y comportamientos relacionados con la cultura de paz, con la intención de que la paz trascienda a través de los mismos alumnos hacia sus familias y a los grupos sociales a los que pertenecen.

La autora nos dice que la mediación educativa, suscita una impresión positiva al buscar una cohesión y pacificación social al trabajar directamente con los alumnos buscando generalizar conductas enmarcadas en la cultura de paz (Vazquez Gutierrez, 2017). Para entender lo anterior se apoya en lo que Fisas (2006) dice sobre cultura de paz, es decir, una superación, reducción o evitación de todo tipo de violencias, con la capacidad y habilidad para transformar los conflictos, como oportunidades creativas, de encuentro, comunicación, cambio, adaptación e intercambio.

Las naciones unidas (1998) definen la cultura de paz como un juego de valores, actitudes, modos de comportamiento y modos de vivir que rechazan la violencia y previenen 
los conflictos abordando sus causas de origen, solucionar problemas mediante el dialogo y la negociación entre individuos, grupos y naciones. Partiendo de lo anterior, es que la Dra. Vázquez plantea como estrategia de pacificación social el formar en valores, convirtiendo estos valores en formas de vida, donde se pueda convivir y solucionar conflictos en lo cotidiano mediante estrategias de pacificación.

Entendiendo lo anterior, damos cuenta como la mediación educativa permite que las acciones de la cultura de paz se interioricen y formalicen como acciones de formación personal e integral de los alumnos, además que facilitan el cumplimiento del paradigma. internacional de la educación para la paz, entendiendo esto como una perspectiva transversal y global (Vazquez Gutiérrez \& Cabello Tijerina, 2018).

La autora define la mediación educativa como una estrategia de la contextualización de la educación para la paz y una educación integral, una formación que engloba todo, compuesta por elementos individuales, recursos sociales, familiares, económicos y materiales. Con miras a formar futuros ciudadanos con habilidades sociales importantes que cuenten con herramientas comunicacionales para resolver sus conflictos de manera pacífica (Vázquez Gutierrez \& García-Longoria Serrano, 2013).

El Dr. Gabriel de Jesús Gorjón Gómez (2015) plantea que la mediación comunitaria como un MSC es un recurso humano, el cual posibilita reconstruir el tejido social, como servicio público de corte universal en el cual tienen una participación los distintos sectores de determinado sitio geográfico, entendiendo que para que uno pueda ejercer su derecho el otro deberá cumplir con su deber, de ser así se logrará una conciencia social permitiendo un cambio de paradigma de cómo se perciben los conflictos comunitarios teniendo efecto en el ámbito intrapersonal social y político. Por lo tanto, la mediación comunitaria como Método de Solución de Conflictos se implementará para transformar las relaciones vecinales, fortaleciendo los derechos humanos y los deberes aludidos en el orden normativo nacional e internacional, cuidando la libertad, igualdad y dignidad humana. en el caso de que exista una falta de conciencia en cuanto al respeto de la libertad de la otra persona.

El Dr. Gabriel plantea que la mediación comunitaria es un recurso social que permite a los integrantes de una comunidad gestionar sus conflictos por ellos mismos, ayudados por un mediador comunitario quien a su vez es miembro de la misma comunidad, quien buscará 
ayudar resolver de manera amigable y sin hacer uso de la justicia ordinaria el conflicto. El mediador a su vez es un voluntario quien gratuitamente presta sus servicios de mediación a la comunidad a la cual pertenece. Deberá conducirse con criterios de equidad, responsabilidad social y garantía de los derechos humanos.

El autor menciona que la mediación es una herramienta del nuevo paradigma de la cultura de paz puesto que promueve el dialogo generando una sensación de justicia. Por lo tanto, la mediación comunitaria al buscar la prevención y la atención de aquellos conflictos barriales de manera armónica y promoviendo el respeto mutuo permiten que se fortalezcan los derechos humanos y de esta manera aspirar a alcanzar la paz.

Uno de los aportes que el Dr. Gabriel Gorjón ha realizado a los MSC, es la difusión de la cultura de estos, así como la capacitación a elementos gubernamentales y abogados, sobre diversos temas como la mediación y sus elementos, las herramientas del mediador, la mediación penal, conciliación y mediación, acuerdos reparatorios, el conflicto, mediación y justicia restaurativa (Gorjón Gómez G. , 2015).

La Dra. Myrna Elia García Barrera (2015) plantea que las nuevas tecnologías de la Información y comunicación tales como las redes sociales, trae muchas ventajas para el ser humano, sin embargo, estas también presentan riesgos tales como el robo de identidad, fraude, ciberdelitos como el cyberbullying, sexting, sexcasting, sextorsión, grooming, cyberstalker, cyberstadking los cuales mediante un método alterno para la solución de conflictos en este caso la mediación como herramienta para la cultura de paz se pueden abordar estos conflictos de manera creativa y diferente a lo jurídico (García Barrera, 2018).

Uno de los aportes que podemos destacar realizado por la Dra. Myrna García es la creación de la teoría del impacto de las TIC's en la vida y la solución de los conflictos, con la utilización de los MSC (García Barrera, 2017). 


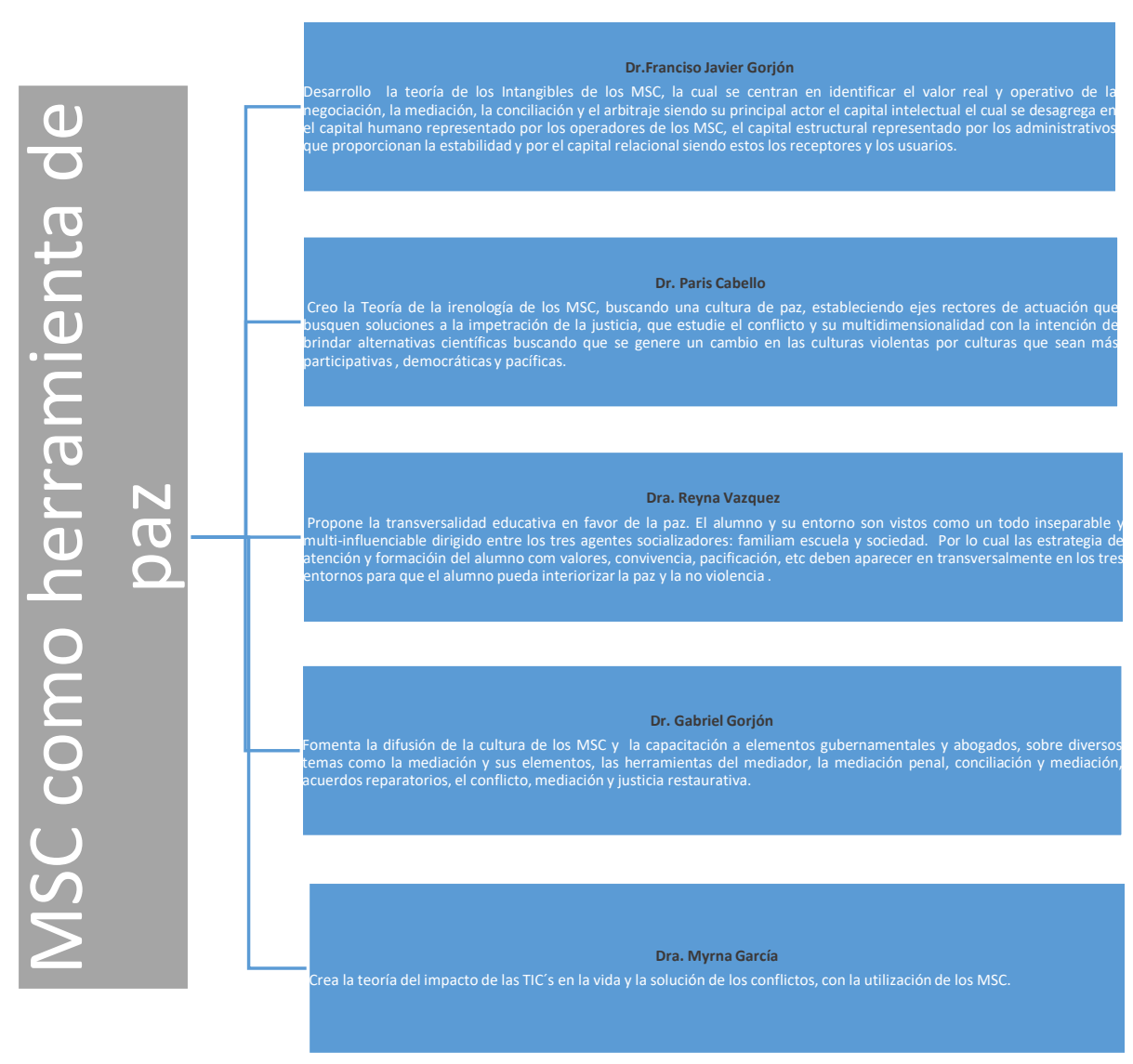

Fuente: Elaboración propia

Por lo tanto, podemos decir que las características con las que cuentan los MSC: arbitraje, mediación, conciliación, negociación como herramientas de paz son que buscan estrategias pacíficas para la solución de los conflictos, cuenta con voluntariedad, promueven la comunicación y colaboración, son educativos y transformadores. Buscan desarrollar actitudes de interés y respeto por los demás, incrementar la capacidad de resolver los conflictos de manera no violenta, fomentando la capacidad para el diálogo, las habilidades comunicativas y la escucha activa. Por lo cual consideramos que los MSC son herramientas en pro de la paz.

Es entonces que los procesos electorales son de suma importancia para las sociedades democráticas como ocurre en nuestro país y que existan diferentes alternativas y medidas para atender su profesionalización, garantizar su efectividad así como facilitar que si se llegase a presentar un conflicto se pueda intervenir de forma pronta y Pacífica, es lo que los 
métodos de solución de conflictos, pueden aportar a el ámbito electoral, para el presente trabaja nos enfocaremos solamente a él capacitador electoral.

Para entender mejor como los MSC potenciarían a los capacitadores electorales, comencemos por reflexionar sobre el tema del conflicto, este se ha abordado desde distintos campos, haciendo un recorrido pertinente para este texto, partimos desde el psicoanálisis en donde el austriaco y neurólogo Sigismund Schlomo Freud (1981) plantea que la interacción de grupos sociales "consiste en un enfrentamiento por choque intencionado entre dos seres o grupos de la misma especie, que manifiestan los unos respecto a los otros, una intención hostil, en general a propósito de un hecho, y que, para mantener, afirmar o restablecer el derecho, tratan de romper la resistencia del otro, eventualmente por el recurso de la violencia, la que puede, llegado el caso, tender al aniquilamiento físico del otro".

Dentro de la psicología la española Jaca Munduate (2006) considera que el conflicto aparece cuando dos personas tienen distintas percepciones respecto a un problema en particular y requieren uno del otro para solucionarlo. En el ámbito de la política el británico/alemán Ralf Gustav Dahrendorf (1990) sostiene que el conflicto es el motor principal del cambio social. En el campo del Derecho el panameño Ricaurte Soler Mendizábal (2002) plantea que el conflicto es una situación en la cual un grupo humano se encuentra en oposición a otro u otros grupos debido a que tienen o persiguen intereses incompatibles; se requiere de una discrepancia cargada de violencia como insultos, peleas, engaños, gritos y agresión.

Siendo pertinente la intervención de los especialistas en formación de MSC para que los capacitadores cuenten con una herramienta más de forma creativa para atender sus avatares dentro de los procesos electorales.

La mediación como uno de los métodos de solución de conflictos cuenta con sus propios principios y utiliza técnicas multidisciplinarias. El mediador es el profesional que ha recibido capacitación previa y específica en esta área, es un profesional de la solución de los conflictos, es decir, no es el quien decide o impone la solución, sino que ayuda a las partes a comunicarse para gestionar su conflicto (Gorjón Gómez F. J., 2019).

Así mismo, el mediador en el procedimiento de mediación promueve el dialogo abierto entre las partes, escucha atentamente la discusión centrada en los intereses, las preocupaciones y los problemas de los mediados con la intención de buscar soluciones 
creativas para arribar a un acuerdo mutuo. El mediador lleva a cabo sus funciones profesionales basado en su experiencia, practica, conocimientos, entrenamiento, inteligencia, técnicas, habilidades y cualidades con las que cuenta (Gorjón Gómez, Riera Adrover, \& Campos Vidal, 2019).

Existe múltiples expertos que como los MSC arrojan beneficios de forma inmediata, uno de ellos es la cultura de paz actualmente se está promoviendo dentro de la SEP con la intención de sus asignaturas, a nivel básico, medio superior y superior. Por otro lado, en algunas escuelas de educación básica existen ejercicios sobre procesos electorales con la finalidad de promover y hacer cultura o promover en los niños y jóvenes la participación ciudadana.

Se puede considerar que estos mismos beneficios servirán para el capacitador electoral ya que es importante que se les pueda transmitir a los funcionarios la capacitación de los MSC, capacitación continua, actualizada, que genere de forma creativa soluciones a los conflictos electorales que estén en su espectro de intervención.

\section{Soluciones creativas a los conflictos electorales}

Para abordar el tema de las soluciones creativas habrá que abordar el tema de la creatividad. A inicios del siglo XIX la creatividad ya no fue considerada como una característica de aquellas personas superdotadas o exclusiva de los llamados genios (Limiñana Gras, 2008), por lo cual comenzó a ser abordada de una manera más dinámica por distintos estudiosos como Freud (1958), Winnicott, Vigotsky, Piaget, entre muchos otros.

Las aportaciones de Freud respecto al proceso creativo se pueden encontrar dispersas en algunos de sus trabajos: La interpretación de los sueños (1900), El chiste y su relación con lo inconciente (1905), los dos principios del acaecer psíquico (1911), El creador literario y el fantaseo (1908). Freud plantea que la producción cultural, así como la creación de obras literarias surgen en el inconsciente del sujeto, originadas desde lo sexual reprimido, es decir, que cuando el ser humano tiene una pulsión que no logra ser satisfecha esta se reprime y es desplazada al inconciente, la sublimación toma este material reprimido transformándolo en material socialmente aceptable, trasladándolo al consciente el cual lo expresa mediante actividades creativas como las artísticas, culturales, literarias, etc. 
Lo que Winnicott (1971), estudia respecto a la creatividad es la aparición, la construcción y la solidez de la experiencia de ser uno mismo, plenamente confiado, es decir, que el ser humano pueda crear, pero sobre todo que tenga la capacidad de vivir de manera creativa mediante experiencias de vida llenas de sentido mediante el juego y la cultura.

La noción de Vigotsky (1990), sobre la creatividad es que surge a partir de una compleja actividad mental sobrepasando el registro y reproducción de la información, considerando que el cerebro además de conservar y reproducir las experiencias pasadas de los seres humanos también combina, transforma y crea utilizando los elementos de las experiencias pasadas poniéndoselas a las nuevas experiencias y a las nuevas conductas. Es decir, la creatividad depende de las experiencias sociales que el ser humano va teniendo a lo largo de su vida, lo cual contribuye a la generación de nuevas maneras de adaptación.

Piaget (1962), entiende el desarrollo de la creatividad desde esquemas de conocimientos y en procesos de acomodación y asimilación. Rogers (1978) plantea que la mayor fuente de creatividad es la autorrealización, por lo cual la creatividad está relacionada con el desarrollo personal, social y emocional, es decir, que el acceso al pensamiento creativo en la vida adulta está ligado con el desarrollo personal, de autonomía y responsabilidad por parte de la persona que se autorrealiza.

Es así, como podemos entender que la creatividad le permite al ser humano mejorar su calidad de vida, haciendo su vida más productiva, aprendiendo a ser competente y original, por lo cual le permite encontrar respuestas a los problemas (Longoría Ramírez, Cantú Hinojosa, \& Ruiz Sepúlveda, 2016).

Existen diferentes tipos de creatividad planteados por Espindola (1996) y Ribot (1901) como la plástica, fluente, filosófica, científica, inventiva, social, entre otras. Son 3 tipos de creatividad las que se pueden desarrollar en los capacitadores electorales desde una formación MSC:

1. Científica: enfocada al ingenio y al talento en la investigación de nuevos conocimientos. Utiliza las ocurrencias en cualquier fase del método, desde la elaboración de hipótesis hasta la generación de opciones para la solución.

2. Inventiva: las ideas, las teorías y los recursos se utilizan de manera talentosa. 
3. Social: se da entre las relaciones humanas, buscando la convivencia pacífica entre los grupos sociales, tiene como objetivo la convivencia y el bienestar buscando lograr el bien común.

Por otro lado, el Dr. Gorjón (2017) plantea la existencia de la creatividad en la mediación como intangible, nos dice que para encontrar soluciones en el proceso de mediación se requiere de opciones las cuales surgen de la creatividad de las personas involucradas en el problema. Apoyando lo anterior, Alés (2011) considera que se pueden crear nuevas formas de solucionar problemas haciendo uso de la creatividad: "El espíritu de la creatividad es el deseo y el impulso de explorar, descubrir, de probar, de experimentar con formas diferentes de manejar y mirar las cosas".

Sin embargo, no todas las personas cuentan con la habilidad creativa para pensar en diversas formas de solucionar un conflicto cuando se enfrentan a uno, por lo cual habrá que desarrollar la creatividad pertinente para cada profesión. En el ámbito MSC se requiere crear un ambiente que posibilite el surgimiento de soluciones a los conflictos, se requiere desarrollar la capacidad de la invención, utilizando el conocimiento previo de la situación y transformarlo para que las personas puedan ver la problemática desde un ángulo distinto, permitiéndoles crear formas distintas de solución a sus conflictos.

El capacitador electoral se puede beneficiar de lo que Ortiz (2007) plantea respecto a la creatividad profesional, sostiene que la creatividad individual es la habilidad que permite solucionar los problemas de manera individual y la creatividad colectiva, es la que favorece los círculos creativos al momento de trabajar en equipo o con un grupo de personas, teniendo como base una actitud abierta, espontanea, con objetivos comunes, autogestión de la acción, autocontrol, retroalimentación y transformación.

\section{Conclusiones:}

La mediación de las crisis electorales es un campo relativamente poco estudiado. Historia reciente sugiere, sin embargo, que a medida que aumenta el número de países que celebran elecciones es aún más probable que ocurra una gran crisis política y de seguridad como resultado de una elección en algún lugar en el mundo anualmente. Las medidas de 
prevención y de capacitación que tomen los países para frenar los actos de violencia en épocas electorales ayudan a la promoción de las democracias en el mundo.

Una manera pacífica de llevar a cabo procesos de mediación en tiempos de campañas electorales son los métodos alternos de solución de conflictos en los que destacan la mediación educativa y comunitaria las cuales fueron mencionadas con anterioridad como lo señala Gorjón (2015) este tipo de mediación es un recurso social que permite a los integrantes de una comunidad gestionar sus conflictos por ellos mismos, ayudados por un mediador comunitario quien a su vez es miembro de la misma comunidad.

En el caso de la mediación educativa es vista como una estrategia de la contextualización de la educación para la paz y una educación integral, una formación que engloba todo. Compuesta por elementos individuales, recursos sociales, familiares, económicos y materiales (Vazquez Gutierrez, 2017).

Los MASC como herramientas para la construcción de herramientas para una cultura de paz, vienen a crear nuevos mecanismos de mediación en la resolución de conflictos electorales. Para encontrar soluciones a los conflictos electorales se requieren de opciones las cuales surgen de la creatividad, por lo cual la formación de capacitadores electorales para la prevención de conflicto conforma una estrategia que puede ayudar a garantizar una solución creativa e inmediata a los conflictos que prevenga la polarización del sistema político y la inestabilidad del Estado.

Sin embargo, la capacitación es más efectiva si todas las instituciones responsables del plan de seguridad electoral del programa de entrenamiento participan en conjunto; el entrenamiento debe llevarse a cabo en las etapas tempranas del proceso electoral para asegurar entrenamientos efectivos de reducción de conflictos (UNPD, 2009) .

Por otro lado, otro enfoque busca cerrar la brecha de comunicación entre ciudadanos y políticos Por lo que se busca la introducción de herramientas digitales que auxilien en el proceso electoral un espacio oficial y que sea también parte de la sociedad civil en donde sea posible que los ciudadanos presenten preguntas y los políticos sean capaces de responder (Sousa \& Agante , 2013). Por lo tanto, las herramientas de participación basadas en internet y redes sociales, son cada vez más importantes en las campañas electorales y si son utilizadas adecuadamente, tienen el potencial de contribuir significativamente a la mediación y prevención de conflictos electorales. 


\section{REFERENCIAS}

Alés, J. (2011). La Creatividad en Mediación: El Modelo Magia y Mediación. Revista Mediato No 0, 26 - 32.

Aparicio, J. (2006). ¿Cómo se financian los partidos políticos en México? . División de Estudios Políticos, $C I D E$, 2-13

http://investigadores.cide.edu/aparicio/FinanPartidosMex_4.pdf.

Arango Durling, V. (2007). Paz social y Cultura de Paz. Panama: Panama Viejo.

Cabello Tijerina, P. A. (2015). La irenología como pilar de la ciencia de la mediación. En F.

J. Gorjón Gómez, \& J. Pesqueira, La ciencia de la mediación (págs. 119-135). México: Tirant lo Blanch.

Cabello Tijerina, P. A. (2016). La mediación como una estrategia de la cultura de paz. En F. P. Arellano Hernández, \& P. A. Cabello Tijerina, Retos y perspectivas de la paz en México. México, México: Tirant lo Blanch.

Cabello Tijerina, P. A. (2016). La mediación policial como política pública de pacificación social. En P. A. Cabello Tijerina, La multidisciplinariedad de la mediación y sus ámbitos de aplicación (págs. 32-49). México: Tirant lo Blanch.

Cabello Tijerina, P. A. (2017). Elementos fundamentales de la cultura de paz. En P. A. Cabello Tijerina, S. E. Carmona Valdés, F. J. Gorjón Gómez, E. Iglesias Ortuño, K. A. Sáenz López, \& R. L. Vázquez Gutiérrez, Cultura de paz (págs. 40-91). México: Patria.

Chacon, M. G. (2018 ). El financiamiento a capacitaciones electorales: de la voluntad politica y otras carencias . Derecho electoral , 107-124 Num 27 file:///C:/Users/arv_8/Downloads/Dialnet-

ElFinanciamientoACapacitacionesElectorales-6523757.pdf.

CICIE. (2015). Talleres internacionales sobre administración electoral. Centro internacional de capacitaciòn y investigaciòn electoral .

Dahrendorf , R. G. (1990). El conflicto social moderno . Madrid: Mondadori.

Espindola Castro, J. L. (1996). Análisis de problemas y toma de decisiones . México: Alambra Mexicana. 
Fisas, V. (2006). Cultura de paz y gestion de conflictos. Barcelona: UNESCO.

Freud, S. (1900). La interpretación de los sueños Tomo IV. Amorrortu editores.

Freud, S. (1905). El chiste y su relación con lo inconciente Tomo VIII. Amorrortu editores.

Freud, S. (1908). El creador literario y el fantaseo. Tomo IX. Amorrortu editores.

Freud, S. (1911). Los dos principios del acaecer psiqiico Tomo XII. Amorrortu editores.

Freud, S. (1958). On creativity and the unconscious . New York: Harper \& Row.

Freud, S. (1981). Psicología de las masas y análisis del Yo. En S. Freud, Obras completas (4 ed.) Tomo III (págs. 2563-2610). Madrid: Biblioteca Nueva.

García Barrera, M. E. (2015). La mediación y los nuevos conflictos en las YIC's: Cyberbullying y sexting. En P. A. Cabello Tijerina, La multidisciplinariedad de la mediación y sus ámbitos de aplicación (págs. 110-128). México: Tirant lo Blanch.

García Barrera, M. E. (2017). Justicia Electónica o Cyber justicia. El tribunal virtual y sus alcances en Nuevo León. México: Acervo de la biblioteca juridica virtual. UNAM. Obtenido de https://archivos.juridicas.unam.mx/www/bjv/libros/6/2958/16.pdf

García Barrera, M. E. (2018). Juzgado sin papel, un paso más de la justicia electónica. IUS, 12(41). Obtenido de http://www.scielo.org.mx/scielo.php?script=sci_arttext\&pid=S187021472018000100133

Gorjón Gómez , G. (2015). La mediación comunitaria como alternativa para fortalecer los derechos humanos. En P. A. Cabello Tijerina, \& J. Moreno Aragon, Diversas miradas, un mismo sentir: comunicación, ciudadanía y paz como retos del siglo XXI. Coahuila: Universidad Autónoma de Coahuila. doi:6074028427, 9786074028423

Gorjón Gómez, F. J. (Julio de 2016). Mediación, ciencia social emergente. Comunitania: International Journal of Social Work and Social Sciences(12), 9-25. doi:http://dx.doi.org/10.5944/comunitania.12.1

Gorjón Gómez, F. J. (2017). Mediación, su valor intangible y efectos operativos. Una vision integradora de los métodos alternos de solución de conflictos. Ciudad de méxico: Tirant lo blanch.

Gorjón Gómez, F. J. (29 de abril de 2019). Mediador y facilitador; El mediador profesional del acuerdo, el facilitador profesional del perdón. Juris Poiesis, 22(28), 229-252. Recuperado el 22 de junio de 2020 
Gorjón Gómez, F. J., \& Sanchez Vázquez, R. (2011). Los MASC como herramientas de paz.

En M. gonzalo Quiroga, F. J. Gorjón Gómez, \& A. Sanchez García, Métodos Alternos de Solución de Conflictos Herramientas de Paz y modernización de la justicia (págs. 29-40). Madrid, España: DYKINSON.

Gorjón Gómez, F. J., Riera Adrover, J. A., \& Campos Vidal, J. F. (2019). Una perspectiva sobre la generación de confianza en España y Latinoamérica. Revista de Mediación, 12(2), 1-10. Recuperado el 21 de junio de 2020

Gorjón Gómez, G. (2015). Mediación: El paradigma Jurídico del siglo XXI. En P. A. Cabello Tijerina, La multidisciplinariedad de la mediación y sus ámbitos de aplicación (págs. 72-85). México: Tirant lo Blanch.

Hernandez, G. Cabello, P. . (2017). Tipologi de los beneficios de la mediacion . Azarbe, 115127.

Kane, S., \& Hayson , N. (2016). Mediaciòn en crisis electorales. International IDEA, 1-28.

Limiñana Gras, R. M. (noviembre de 2008). Cuando crear es algo más que un juego: creatividad, fantasía e imaginación en los jóvenes. Cuadernos de la Facultad de Humanidades y Ciencias Sociales(35), 39-43.

Longoría Ramírez, R., Cantú Hinojosa, I. L., \& Ruiz Sepúlveda, J. D. (2016). Pensamieto creativo. México: Patria.

Munduate Jaca, L. \&. (2006). Gestion del conflicto, negociación y mediación. Madrid: Piramide.

Nassif, A. A. (2007). El retorno del conflicto. Elecciones y polarización política en México. Desacatos.

Ortiz Ocaña, A. L. (2007). La educación y el desarrollo de la creatividad: Un reto en la formación de profesionales .

Pérez , J., \& Gardey, A. (2013). Definición de paz. Obtenido de https://definicion.de/paz/

Piaget, J. (1962). Play, dreams and imagination in childhood. New York: Morton.

Ribot, T. (1901). Ensayo sobre la imaginación creadora. Madrid: Victoriano Sárez.

Ricaurte, S. M. (2002). El arbitraje, la conciliación y la mediación. Métodos alternos de solución de conflictos. Panama: Universal Books.

Rogers, C. (1978). Hacia una teoría de la creatividad. Madrid, Narcea: Th. Roberts (Ed). 
Shale, V., \& Gerenge , R. (2017). Mediación Electoral en la Democracia de Republica de Congo. Conflict trends.

Sousa, A., \& Agante , P. (2013). Modelo de mediación digital para la participación pública directa en períodos electorales: ¿qué importancia tienen los medios? conference paper

Thompson, J. (2012). La labor educativa en el marco de las competencias de los organismos electorales en America Latina . derecho electoral, 10.

unidas, A. g. (15 de enero de 1998). resolución adaptada por la asamblea general 52/13 Cultura de paz.

UNPD. (2009). Elecciones y prevencion de conflictos . Bureau for Development Policy.

Valdez , A., \& Huerta , D. (2018). El financiamiento de los partidos políticos y la confianza $\begin{array}{llllll}\text { ciudadana en } & \text { México. Intersticios } & \text { sociales, }\end{array}$ http://www.scielo.org.mx/scielo.php?script=sci_arttext\&pid=S2007$49642018000100309 \& \operatorname{lng}=$ es\&nrm=iso.

Vázquez Gutierrez, R. L. (2015). Mediación educativa como instrumento de pacificación social. En P. A. Cabello Tijerina, La multidisciplinariedad de la mediación y sus ámbitos de aplicación (págs. 55-68). México: Tirant lo Blanch.

Vazquez Gutierrez, R. L. (2017). Contextualización de la educación para la paz en el ámbito universitario. En P. A. Cabello Tijerina, S. E. Carmona Valdés, F. J. Gorjón Gómez, E. Iglesias Ortuño, K. A. Sáenz López, \& R. L. Vázquez Gutiérrez, Cultura de paz (págs. 92-111). México: Patria.

Vazquez Gutiérrez, R. L., \& Cabello Tijerina, P. A. (2018). Cultura y educación para la paz. Una perspectiva transversal. México: tirant lo Blanch.

Vázquez Gutierrez, R. L., \& García-Longoria Serrano, M. P. (enero de 2013). La mediación escolar y la habilidades sociales en los estudiantes de educación secundaria. Un estudio en institutos de la región de Murcia. Comunitania(5), 113-136.

Vigotsky, L. (1990). Imagination and creativity in the adolescent. Soviet Psychology, 84-96. Winnicott, D. (1971). Clínica Psicoanalítica infantil. Bs.s: Paidos. 